\title{
Clinical outcomes and survival differences between primary, secondary and concomitants carcinoma in situ of urinary bladder treated with BCG immunotherapy
}

\author{
Radosław Piszczek ${ }^{1 \#}$, Wojciech Krajewski ${ }^{2 \#}$, Bartosz Małkiewicz ${ }^{2}$, Piotr Krajewski ${ }^{2}$, Andrzej Tukiendorf ${ }^{3}$, \\ Romuald Zdrojowy ${ }^{2}$, Anna Kołodziej ${ }^{2}$ \\ ${ }^{1}$ Department of Urology and Oncologic Urology, Lowersilesian Specialistic Hospital, Wroclaw, Poland; ${ }^{2}$ Department of Urology and Oncological \\ Urology, ${ }^{3}$ Department of Public Health, Wrocław Medical University, Wrocław, Poland \\ Contributions: (I) Conception and design: W Krajewski; (II) Administrative support: None; (III) Provision of study materials or patients: A Kołodziej; \\ (IV) Collection and assembly of data: P Krajewski; (V) Data analysis and interpretation: B Małkiewicz, A Tukiendorf; (VI) Manuscript writing: All \\ authors; (VII) Final approval of manuscript: All authors. \\ \#These authors contributed equally to this work. \\ Correspondence to: Wojciech Krajewski. Department of Urology and Oncological Urology, Wroclaw Medical University, Borowska 213, Wroclaw, \\ Poland. Email: wk@softstar.pl.
}

Background: The carcinoma in situ (CIS) of urinary bladder is a flat, high-grade and aggressive manifestation of urothelial cancer which carries a great risk for progression to muscle invasive disease and metastatic spread. The aim of the study was to analyse the clinical history and survival differences between primary, secondary and concomitants CIS of the bladder.

Methods: We analysed a database of 301 patients who were treated between 1998 and 2017 in university BCG outpatient department. Primary, secondary and concomitants CIS cases were included in the study with minimum follow-up of 12 months. Recurrence-free survival (RSF), progression-free survival (PFS) and cancer specific survival (CSS) were analysed by Kaplan-Meier curves and log-rank test. Additionally, Cox regression models were performed.

Results: Seventy-two patients with primary, 58 with secondary and 171 with CIS concomitants were analysed. The patients were followed from mean 61,9 months. RFS rates at 5-years follow-up were 54.9\%, $55.3 \%$ and $60.4 \%$ for primary, concomitants and secondary CIS (P>0.05). PFS rates at 5-years follow-up were $65.8 \%, 72.1 \%$ and $77 \%$ for primary, concomitants and secondary CIS $(\mathrm{P}>0.05)$. CSS rates at 5 -years follow-up were $83.1 \%, 81.9 \%$ and $90.1 \%$ for primary, concomitants and secondary CIS $(\mathrm{P}>0.05)$. There were no statistically significant differences between any CIS subtype in any of analysed endpoints in multivariate analysis. Female gender was associated with worse RFS and the instillation number was positively associated with all analysed survival rates. On contrary, age was not statistically related to RFS, PFS nor CSS.

Conclusions: In this study it was shown that when compared with secondary and concomitants CIS, primary CIS presented the worst RFS and PFS, however, the differences between subgroups were not statistically significant.

Keywords: Bladder cancer (BC); carcinoma in situ (CIS); recurrence; progression

Submitted Sep 07, 2019. Accepted for publication Feb 14, 2020.

doi: $10.21037 /$ tau.2020.03.40

View this article at: http://dx.doi.org/10.21037/tau.2020.03.40 


\section{Introduction}

After prostate cancer, bladder cancer (BC) is the second most common malignancy of the genitourinary system. According to European Association of Urology (EAU) it is the seventh most frequent carcinoma in men in the developed countries (1).

At the time of primary diagnosis, approximately $75 \%$ of patients diagnosed with urothelial cell carcinoma (UCC) are at non-muscle-invasive stage (NMIBC; non-muscle invasive bladder cancer) comprising $\mathrm{Ta}, \mathrm{T} 1$ and carcinoma in situ (CIS) stages. The CIS is a flat, high-grade and aggressive manifestation of UCC which carries a great risk for progression to muscle invasive disease and metastatic spread (2).

Despite the fact, that epidemiological data about all BCs is generally reliable, the incidence of CIS in the bladder is ambiguous. Some papers state that only $<2 \%$ of patients diagnosed with NIMBC present CIS (3). On the other hand, in the study by Millán-Rodríguez et al., where more than 1,500 patients with primary NMIBC underwent multiple bladder biopsies (mapping), authors proved that $19 \%$ of all cases were associated with CIS (4). Additionally, the clinical history of CIS and differences in survival of patients with various CIS subtypes are not well described in the literature. Available studies present small populations with mixed clinical stages observed for short periods of time.

The aim of the study was to analyse the clinical history and survival differences between primary, secondary and concomitants CIS of urinary bladder.

\section{Methods}

We analysed a database of 301 patients who were treated between 1998 and 2017 in University Centre BCG outpatient department. Primary, secondary and concomitants CIS cases were included in the study with minimum follow-up of 12 months. Most of the specimens were originally evaluated by the team of three dedicated uropathologists. In the case of doubtful test results from other centres, specimens were reviewed and verified by our team. Whole BCG immunotherapy schedule consisted of 27 instillations divided into introducing course and seven maintenance courses. Introducing course was composed of six weekly given instillations. Maintenance courses were comprised of three weekly given instillation administered after 3, 6, 12, 18, 24, 30 and 36 months. Patients with at least 7 instillations (introduction course and any maintenance) were included in the analysis. All patients were treated with induction and maintenance immunotherapy schedules using TICE, RIVM and Moreau BCG strains. Cystoscopy (CS) with urine cytology was performed every 3-6 months during first two years following TURB and then every 6 months. After five years of follow-up CS was performed annually. Upper tract imaging (IVU or CT scan) was performed every 2 years and also in case of unexplained positive cytology and upper tract symptoms.

Primary CIS was defined as an isolated CIS with no previous or concurrent papillary tumour. Concomitants CIS was defined as CIS detected simultaneously with papillary tumour. Finally, secondary CIS was defined as CIS lesion detected during the follow-up of patients with a previous papillary tumour. A recurrence was defined as a reoccurrence of tumour of any stage and grade confirmed by TURBT and histologic or cytological assessment. Progression was defined tumour relapse at tumour stage T2 or higher in the bladder or stromal invasion of the prostatic urethra.

\section{Statistical analysis}

Group comparison was analysed with ANOVA test. The variations of frequencies were tested using $\chi^{2}$. Times to events were calculated taking the date of initiating BCG as time zero. Recurrence-free survival (RSF), progression-free survival (PFS), cancer specific survival (CSS) were estimated using the Kaplan-Meier curves. Patients without an event or death before an event were censored at the last date of follow-up. Differences in survival rates were assessed using a log-rank test. Cox regression models were also performed. Analyses were conducted with SPSS software (version 22.0, SPSS Inc., Chicago, III., USA). P values of $<0.05$ were regarded significant.

\section{Results}

The study included 301 patients (age 67.2 $\pm 8.7,55$ Female/246 Male) diagnosed with CIS and treated between 1998 and 2017. From all patients included in the analysis 171 had CIS concomitants to exophytic tumour, 72 primary and 58 secondary CIS. Basic tumour characteristics are shown in Table 1. Total 59 patients completed whole BCG regimen (27 installations) and average number of installations was 16.37 .

The patients were followed from mean 61.9 months. The 
Table 1 The patients baseline characteristics (Pearson chi2 and ANOVA test p-values of the differences between the groups)

\begin{tabular}{|c|c|c|c|c|c|}
\hline & $\begin{array}{l}\text { All cases } \\
n=301,(\%)\end{array}$ & $\begin{array}{l}\text { Primary CIS } \\
\mathrm{n}=72(23.9 \%)\end{array}$ & $\begin{array}{l}\text { Secondary CIS } \\
n=58(19.3 \%)\end{array}$ & $\begin{array}{l}\text { Concomitants CIS } \\
n=171(56.8 \%)\end{array}$ & $P$ value \\
\hline Gender (M/F) & $246(81.7) / 55(18.3)$ & $59(81.9) / 13(18.1)$ & $48(82.8) / 10(17.2)$ & $139(81.3) / 32(18.7)$ & 0.968 \\
\hline Observation time months (mean; SD) & $61.9 ; 50.1$ & $65.3 ; 52.0$ & $55.2 ; 30.5$ & $62.8 ; 55.7$ & 0.504 \\
\hline Cancer specific death & $44(14.6)$ & $13(18.1)$ & $5(8.6)$ & $26(15.2)$ & 0.155 \\
\hline $\begin{array}{l}\text { Number of papillary tumours } \\
\text { (solitary/multiple/unk) }\end{array}$ & & & & $68(39.8) / 90(52.6) / 13(7.6)$ & \\
\hline $\begin{array}{l}\text { Papillary tumour size } \\
(<3 \mathrm{~cm} / 3 \mathrm{~cm} / \mathrm{unk})\end{array}$ & & & & $\begin{array}{c}48(28.1) / 109(63.7) / 14 \\
(8.2)\end{array}$ & \\
\hline $\begin{array}{l}\text { Papillary tumour grade WHO1973 } \\
(1 / 2 / 3)\end{array}$ & & & & $\begin{array}{c}25(14,6) / 80(46.8) / 66 \\
(38.6)\end{array}$ & \\
\hline
\end{tabular}

The value of adjusted $\mathrm{P}<0.05$ was considered statistically significant. CIS, carcinoma in situ; M, male; F, female; SD, standard deviation; unk, unknown.

recurrence was observed in 113 (35.5\%) with 12 months' median time to the first recurrence. Progression of the cancer was observed in 78 pts $(25.9 \%)$ and cancer specific death occurred in 44 of patients $(14.6 \%)$.

RSF rates at 5-years follow-up were $54.9 \%, 55.3 \%$ and $60.4 \%$ for primary, concomitants and secondary CIS groups, respectively. Despite some visible trends on Kaplan curves (Figure 1), the results of log-rank test were statistically non-significant $(\mathrm{P}>0.05)$ for both general and pair analysis (Table 2).

PFS rates at 5-years follow-up were $65.8 \%, 72.1 \%$ and $77 \%$ for primary, concomitants and secondary CIS groups, respectively (Figure 2). Once more, log-rank results were not statistically significant.

Finally, CSS rates at 5 -years follow-up were $83.1 \%$, $81.9 \%$ and $90.1 \%$ for primary, concomitants and secondary CIS groups, respectively. Similarly, log-rank results were not statistically significant.

The results of cox regression analysis are presented in Table 3. Despite the fact, that there were no statistically significant associations of CIS subtype with any of analysed end points, we have found that female gender was associated with worse RFS and the instillation number was associated positively with all analysed survival rates. On contrary, age was not statistically related to RFS, PFS nor to CSS.

\section{Discussion}

In this study, survival differences between primary, secondary and concomitants CIS of urinary bladder were analysed in population of 301 patients. It was shown that primary CIS presented the worst RFS and PFS, however, the differences between subgroups were not statistically significant. Also, there were no statistically significant associations of CIS subtype with any of analysed end points in cox regression analysis. Interestingly, we have found that female gender was associated with worse RFS and that patient age was not statistically related to RFS, PFS nor CSS.

The impact of CIS on oncological outcomes of NMIBC was widely discussed in the literature for many years. In both EORTC (European Organization for Research and Treatment of Cancer) and CUETO (Spanish Urological Club for Oncological Treatment) prognostic nomograms, CIS are incorporated as aggravating factor, yet, with smaller negative influence for BCG treated population $(5,6)$. In any case, the studies analyzing clinical course of the CIS prove that this is aggressive entity characterized by high disease progression risk (7). 


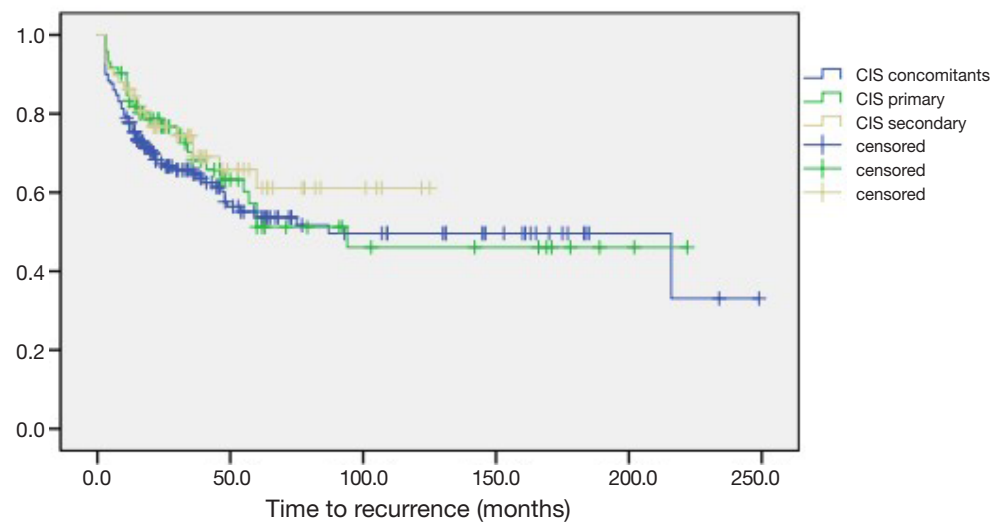

Figure 1 Recurrence-free survival for the concomitants, primary and secondary CIS groups (log-rank test $\mathrm{P}=0.430)$. CIS, carcinoma in situ.

Table 2 P-values of Log-rank survival analysis according to tumour subtype

\begin{tabular}{lcccc}
\hline & All cases & Concomitants vs. secondary & Concomitants vs. primary & Secondary vs. primary \\
\hline RFS & 0.430 & 0.222 & 0.509 & 0.536 \\
PFS & 0.199 & 0.374 & 0.256 & 0.072 \\
CSS & 0.344 & 0.149 & 0.953 & 0.228 \\
\hline
\end{tabular}

The value of adjusted $\mathrm{P}<0.05$ was considered statistically significant. RFS, recurrence-free survival; PFS progression-free survival; CSS, cancer-specific survival.

The subject of differences in survival between various CIS subgroups was addressed previously with conflicting results. The available studies are burdened with retrospective nature, low cases number and heterogenicity of definitions and treatment regiments.

Chad et al. found that primary CIS carries a higher risk of progression to both $\mathrm{T} 1$ and $\mathrm{T} 2$ stages than secondary CIS. Despite somehow comparable results in both our and Chad's paper, it ought to be emphasized that definitions adopted in the studies were different. In Chad's study, progression was defined as tumour relapse at tumour stage T1 (T2 in our study) and secondary CIS was defined as diagnosed concomitantly with or after a prior papillary Ta tumour (Ta and T1 in our study) (8). In recent study assessing long follow-up of patients treated with BCG monotherapy versus alternating therapy with mitomycin C and BCG by Kaasinen et al. it was shown that primary CIS showed the worst RFS when compared with concomitants and secondary cases. In contrast to recurrence, disease progression was most likely in patients with concomitant CIS and least likely in those with primary CIS (9). Similarly, in a paper by Kim et al., a poorer prognosis for progression was shown for primary CIS and T1 concomitants CIS in a group of 254 patients with either primary or concurrent CIS (10).

On the other hand, in the other paper describing 30906 EORTC trial results, no differences in complete response rates between primary, secondary and concurrent CIS were observed (11). Also, van Gils-Gielen et al. analyzed in randomized fashion a group of 52 patients with CIS and found no disparities in the risk of recurrence and progression between CIS alone and CIS associated with papillary tumors. Yet, the patients were treated with various therapeutic regimens including intravesical mitomycin and BCG (12). Takenaka et al. reported a cohort of 185 CIS patients treated with weekly, 8-week BCG instillations and showed no difference between CIS subtypes in terms of risk of recurrence and progression (13). Similar results were achieved in a study by Gofrit et al. who analyzed a sample of 104 patients with CIS , showing no significant difference between primary and concomitant CIS (14).

In contrary, in the study on 93 patients by Hayakawa et $a l$. it was found that secondary CIS recurred significantly more in 5-year period when compared with concurrent and primary CIS. Progression free-survival has not been reported in that study, yet, only $74.2 \%$ of patients 


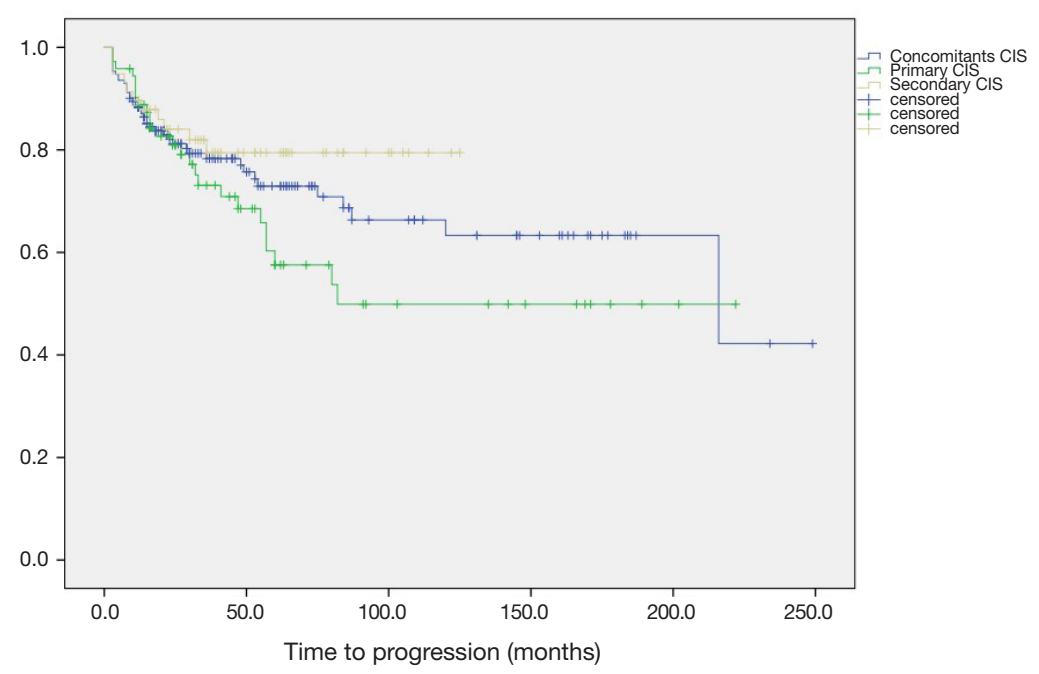

Figure 2 Progression-free survival for the concomitants, primary and secondary CIS groups (log-rank test P=0.199). CIS, carcinoma in situ.

underwent BCG therapy (15). In other study, Griffiths et al. evaluated the outcome of primary CIS and CIS associated with Ta or T1 UCC of the bladder. They found that primary CIS had good prognosis, while coexistence of CIS and T1 lesion carries a significantly greater risk of progression. It has to be highlighted that only introduction courses were used in that study (16).

Despite the fact that we did not analyze in our study the prognostic value of CIS extent in the bladder, it is worth mentioning that some papers suggested that focal lesions are associated with better prognosis, while the others found no prognostic significance $(10,12,15)$. Because of that, it is still unclear whether the greater extent of CIS lesions is associated with worse prognosis and poorer treatment outcomes, however, with improved cystoscopic imaging, the distinction between focal and diffuse CIS may become more meaningful in terms of prognosis in the future.

The results of our study, showing inferior RFS and PFS rates for primary CIS, although not statistically significant, are consistent with results reported by other authors suggesting that primary CIS is more aggressive disease. Despite the heterogenicity of available data, the subclassification of CIS may be helpful in identifying patients with worse prognosis, who can be treated radically before progression to muscle-invasive disease. Nevertheless, further studies are required to demonstrate significant differences in outcomes between CIS subtypes and potential influence of that distinction on clinical management of the disease.

The median age at BC diagnosis is above 70 . Also, it is well known that immune response deteriorates with rising age $(17,18)$. Hence, it may be supposed that BCG response would be weaker in older people. On the other hand, it was shown before, that the $\mathrm{BC}$ may be more aggressive in younger individuals. What is worth highlighting, it was observed in our study that despite older patients received less instillations, there were no differences in survival analysis. Yet, as this was an side observation, the conclusions should be drawn carefully.

Differences in BC survival between genders were discussed widely in the literature (19-21). The general conclusion is that survival rates of females tend to be worse than males. In this study, female gender was associated with worse RFS, yet, not with worse PFS and CSS. Once more, the conclusions should be drawn carefully.

Our study had some limitations. Firstly, it was a retrospective analysis with long period of the observation and possible cohort effect and selection bias. Secondly, despite the abundant number of all CIS cases, the somehow scarce number of endpoints after division may cause the low power of statistical analysis and bias the results. It is particularly true in progression and mortality analysis.

\section{Conclusions}

In this study it was shown that when compared with secondary and concomitants CIS, primary CIS presented the worst RFS and PFS, however, the differences between subgroups were not statistically significant. More studies regarding this issue are required, as there is still lack of 
Table 3 Multivariable analysis for prognostic factors for RFS, PFS and CSS

\begin{tabular}{|c|c|c|c|c|c|c|c|c|c|}
\hline Variable & \multicolumn{3}{|c|}{ Recurrence-free survival } & \multicolumn{3}{|c|}{ Progression-free survival } & \multicolumn{3}{|c|}{ Cancer-specific survival } \\
\hline CIS concomitant (ref) & 0.211 & - & - & 0.431 & - & - & 0.241 & - & - \\
\hline Primary & 0.214 & 1.39 & $0.83-2.34$ & 0.212 & 1.53 & $0.76-2.99$ & 0.099 & 2.27 & $0.87-5.95$ \\
\hline Secondary & 0.247 & 0.98 & $0.54-1.79$ & 0.258 & 1.52 & $0.75-3.13$ & 0.243 & 1.86 & $0.66-5.24$ \\
\hline Gender (female vs. male) & 0.037 & 1.82 & $1.04-3.19$ & 0.075 & 1.88 & $0.94-3.78$ & 0.432 & 1.47 & $0.57-3.74$ \\
\hline Instillation number & 0 & 0.92 & $0.89-0.95$ & 0 & 0.89 & $0.87-0.93$ & 0 & 0.89 & $0.85-0.93$ \\
\hline
\end{tabular}

The value of adjusted $\mathrm{P}<0.05$ was considered statistically significant. HR, Hazard ratio; $95 \% \mathrm{Cl}, 95 \%$ confidence interval; CIS, carcinoma in situ; HR $>1$ worse outcome for the bolded option, $\mathrm{HR}<1$ better outcome for the bolded option.

good quality evidence supporting the use of CIS subclassification in clinical practice.

\section{Acknowledgments}

Funding: None.

\section{Footnote}

Conflicts of Interest: All authors have completed the ICMJE uniform disclosure form (available at http://dx.doi. org/10.21037/tau.2020.03.40). The authors have no conflicts of interest to declare.

Ethical Statement: The authors are accountable for all aspects of the work in ensuring that questions related to the accuracy or integrity of any part of the work are appropriately investigated and resolved. The study was approved by the Ethics Committee of Lowersilesian Specialistic Hospital, Number of ethical approval: 459/2016. Informed consent was obtained from all individual participants at the time of their BCG therapy onset stating that in future their data may be included in the scientific studies.

Open Access Statement: This is an Open Access article distributed in accordance with the Creative Commons Attribution-NonCommercial-NoDerivs 4.0 International License (CC BY-NC-ND 4.0), which permits the noncommercial replication and distribution of the article with the strict proviso that no changes or edits are made and the original work is properly cited (including links to both the formal publication through the relevant DOI and the license).
See: https://creativecommons.org/licenses/by-nc-nd/4.0/.

\section{References}

1. Babjuk M, Bohle A, Burger M, et al. EAU Guidelines on Non-Muscle-invasive Urothelial Carcinoma of the Bladder: Update 2016. Eur Urol 2017;71:447-61.

2. Solsona E, Madero R, Chantada V, et al. Sequential combination of mitomycin $\mathrm{C}$ plus bacillus CalmetteGuerin (BCG) is more effective but more toxic than BCG alone in patients with non-muscle-invasive bladder cancer in intermediate- and high-risk patients: final outcome of CUETO 93009, a randomized prospective trial. Eur Urol 2015;67:508-16.

3. Chavan S, Bray F, Lortet-Tieulent J, et al. International variations in bladder cancer incidence and mortality. Eur Urol 2014;66:59-73.

4. Millán-Rodríguez F, Chechile-Toniolo G, Salvador-Bayarri $\mathrm{J}$, et al. Multivariate analysis of the prognostic factors of primary superficial bladder cancer. J Urol 2000;163:73-8.

5. Sylvester RJ, van der Meijden AP, Oosterlinck W, et al. Predicting recurrence and progression in individual patients with stage Ta T1 bladder cancer using EORTC risk tables: a combined analysis of 2596 patients from seven EORTC trials. Eur Urol. 2006;49:466-5; discussion 475-7.

6. Fernandez-Gomez J, Madero R, Solsona E, et al. Predicting nonmuscle invasive bladder cancer recurrence and progression in patients treated with bacillus CalmetteGuerin:the CUETO scoring model. J Urol 2009;182:2195203.

7. Chade DC, Shariat SF, Godoy G, et al. Clinical outcomes of primary bladder carcinoma in situ in a contemporary 
series. J Urol 2010;184:74-80.

8. Chade DC, Shariat SF, Adamy A, et al. Clinical outcome of primary versus secondary bladder carcinoma in situ. J Urol 2010;184:464-9.

9. Kaasinen E, Wijkstrom H, Rintala E, et al. Seventeenyear follow-up of the prospective randomized Nordic CIS study: BCG monotherapy versus alternating therapy with mitomycin $\mathrm{C}$ and BCG in patients with carcinoma in situ of the urinary bladder. Scand J Urol 2016;50:360-8.

10. Kim SJ, You D, Jeong IG, et al. Prognosis of carcinoma in situ according to the presence of papillary bladder tumors after bacillus Calmette-Guerin immunotherapy. J Cancer Res Clin Oncol 2019;145:2131-40.

11. de Reijke TM, Kurth KH, Sylvester RJ, et al. Bacillus Calmette-Guerin versus epirubicin for primary, secondary or concurrent carcinoma in situ of the bladder: results of a European Organization for the Research and Treatment of Cancer--Genito-Urinary Group Phase III Trial (30906). J Urol 2005;173:405-9.

12. van Gils-Gielen RJ, Witjes WP, Caris CT, et al. Risk factors in carcinoma in situ of the urinary bladder. Dutch South East Cooperative Urological Group. Urology 1995;45:581-6.

13. Takenaka A, Yamada Y, Miyake H, et al. Clinical outcomes of bacillus Calmette-Guerin instillation therapy for carcinoma in situ of urinary bladder. Int J Urol 2008;15:309-13.

Cite this article as: Piszczek R, Krajewski W, Małkiewicz B, Krajewski P, Tukiendorf A, Zdrojowy R, Kołodziej A. Clinical outcomes and survival differences between primary, secondary and concomitants carcinoma in situ of urinary bladder treated with BCG immunotherapy. Transl Androl Urol 2020;9(3):13381344. doi:10.21037/tau.2020.03.40
14. Gofrit ON, Pode D, Pizov G, et al. The natural history of bladder carcinoma in situ after initial response to bacillus Calmette-Guerin immunotherapy. Urol Oncol 2009;27:258-62.

15. Hayakawa N, Kikuchi E, Mikami S, et al. The clinical impact of the classification of carcinoma in situ on tumor recurrence and their clinical course in patients with bladder tumor. Jpn J Clin Oncol 2011;41:424-9.

16. Griffiths TR, Charlton M, Neal DE, et al. Treatment of carcinoma in situ with intravesical bacillus CalmetteGuerin without maintenance. J Urol 2002;167:2408-12.

17. DeVeale B, Brummel T, Seroude L. Immunity and aging: the enemy within? Aging Cell 2004;3:195-208.

18. Pfister G, Savino W. Can the immune system still be efficient in the elderly? An immunological and immunoendocrine therapeutic perspective. Neuroimmunomodulation 2008;15:351-64.

19. Andreassen BK, Grimsrud TK, Haug ES. Bladder cancer survival: Women better off in the long run. Eur J Cancer 2018;95:52-8.

20. Richters A, Dickman PW, Witjes JA, et al. Bladder cancer survival: Women only fare worse in the first two years after diagnosis. Urol Oncol 2019;37:853-61.

21. Radkiewicz C, Edgren G, Johansson ALV, et al. Sex Differences in Urothelial Bladder Cancer Survival. Clin Genitourin Cancer 2020;18:26-34.e6. 